\title{
Conhecimento dos profissionais de saúde acerca do núcleo de apoío à saúde da família
}

\author{
Knowledge of health professionals about the family health support center
}

\author{
Conocimiento de los profesionales de la salud sobre el centro de apoyo a la salud familiar
}

\begin{abstract}
RESUMO
Objetivo: Verificar na literatura científica o conhecimento dos profissionais de saúde acerca do Núcleo de Apoio à Saúde da Família (NASF). Método: Trata-se de uma revisão integrativa. Realizou-se a busca por artigos; publicados nos últimos dez anos (2009 a 2019); nos idiomas português, inglês e espanhol; disponiveis na íntegra. Foram consultadas as bases: BVS, MEDLINE, LILACS; e Biblioteca Virtual: SciELO. Os dados foram organizados e apresentados em figuras e tabelas. Resultados: Dos 128 estudos encontrados, 12 estavam disponíveis na BDENF; 1 na MEDLINE; 69 na LILACS; e 46 na SciELO; contudo, após a leitura permaneceram apenas 13 estudos. Após a leitura os artigos foram categorizados em recortes temáticos, classificando o conhecimento produzido. Conclusão: Constatou-se que os profissionais de saúde compreendem a estrutura do NASF, ainda que com algum déficit de informação, declarando o serviço como de extrema importância, apresentando satisfação acerca das atividades realizadas, categorizando como serviço alcançável pela população.
\end{abstract}

DESCRITORES: Estratégia Saúde da Família; Equipe de Assistência ao Paciente; Atenção Primária à Saúde.

\section{ABSTRACT}

Objective: To verify in the scientific literature the knowledge of health professionals about the Family Health Support Center (NASF). Method: This is an integrative review. The search for articles was carried out; published in the last ten years (2009 to 2019); in Portuguese, English and Spanish; available in full. The following databases were consulted: BVS, MEDLINE, LILACS; and Virtual Library: SciELO. Data were organized and presented in figures and tables. Results: Of the 128 studies found, 12 were available in BDENF; 1 in MEDLINE; 69 in LILACS; and 46 in SciELO; however, after reading, only 13 studies remained. After reading, the articles were categorized into thematic sections, classifying the knowledge produced. Conclusion: It was found that health professionals understand the structure of the NASF, although with some information deficit, declaring the service as extremely important, showing satisfaction about the activities performed, categorizing it as a service reachable by the population.

DESCRIPTORS: Family Health Strategy; Patient Care Team; Primary Health Care.

\section{RESUMEN}

Objetivo: Verificar en la literatura científica el conocimiento de los profesionales de la salud sobre el Centro de Apoyo a la Salud de la Familia (NASF). Método: Esta es una revisión integradora. Se realizó la búsqueda de artículos; publicado en los últimos diez años (2009 a 2019); en portugués, inglés y español; disponible en su totalidad. Se consultaron las siguientes bases de datos: BVS, MEDLINE, LILACS; y Biblioteca Virtual: SciELO. Los datos se organizaron y presentaron en figuras y tablas. Resultados: De los 128 estudios encontrados, 12 estaban disponibles en BDENF; 1 en MEDLINE; 69 en LILACS; y 46 en SciELO; sin embargo, después de la lectura, solo quedaron 13 estudios. Después de la lectura, los artículos se categorizaron en secciones temáticas, clasificando el conocimiento producido. Conclusión: Se encontró que los profesionales de la salud comprenden la estructura de la NASF, aunque con cierto déficit de información, declarando el servicio de suma importancia, mostrando satisfacción por las actividades realizadas, categorizándolo como un servicio accesible para la población.

DESCRIPTORES: : Estrategia de Salud de la Familia; Equipo de Atención al Paciente; Atención Primaria de Salud.

RECEIVED: 08/06/21 APPROVED: 14/06/21

\section{Giovana Maria Correia de Siqueira}

Especialista em Saúde Coletiva com ênfase em Saúde da Família pela Faculdade Redentor - Instituto de Desenvolvimento Educacional (IDE). Recife, Pernambuco (PE), Brasil.

ORCID ID: https://orcid.org/0000-0002-9681-8397

\section{Liniker Scolfild Rodrigues da Silva}

Especialista em Saúde Coletiva na modalidade Residência pela Faculdade de Ciências Médicas (FCM)/Universidade de Pernambuco (UPE). Especialista em Saúde Pública com ênfase a Saúde da Família pela Faculdade INESP (Instituto Nacional de Ensino e Pesquisa). Recife, Pernambuco (PE), Brasil.

ORCID ID: https://orcid.org/0000-0003-3710-851X 


\section{Yanna Georgia Crispiniano Ferreira da Silva}

Licenciatura em Enfermagem pela Universidade Católica Portuguesa. Lisboa, Portugal.

ORCID ID: https://orcid.org/0000-0001-9168-3676

\section{Pedro Augusto Maia Gomes}

Discente do Curso de Bacharel em Medicina pela Faculdade Pernambucana de Saúde (FPS). Recife, Pernambuco (PE), Brasil. ORCID ID: https://orcid.org/0000-0002-3765-0897

\section{Priscila Bruna Pereira de Matos}

Enfermeira (egressa), Centro Universitário São Miguel (UNISAOMIGUEL). Recife, Pernambuco (PE), Brasil.

ORCID ID: https://orcid.org/0000-0002-9034-475X

\section{Mannuelle dos Santos Ferreira}

Especialista em Enfermagem Obstétrica pelo Centro Universitário São Miguel (UNISAOMIGUEL). Recife, Pernambuco (PE), Brasil.

ORCID ID: https://orcid.org/0000-0002-0129-5889

\section{INTRODUÇÃO}

A Estratégia Saúde da Família (ESF) foi idealizada visando expandir o ingresso da população e propiciar uma atuação focada nos territórios e nas residências. Dando início com a atuação de equipes mínimas, muito próximas das equipes que já vinham operando na $A B$, porém, se tornou perceptível a indispensabilidade de outros profissionais, para se pudesse ocorrer uma expansão dos olhares e maior possibilidade de se aproximar do princípio da integralidade (1).

O Ministério da Saúde (MS), mediante a portaria GM n. 154, de 24 de janeiro de 2008, desenvolveu o Núcleo de Apoio à Saúde da Família (NASF), com o propósito de reestruturar a assistência à saúde com relação à atenção básica e contribuir na qualificação do Sistema Único de Saúde (SUS). Desta forma, o NASF visa colaborar com os profissionais da $\mathrm{AB}$, possuindo como princípio básico um trabalho interdisciplinar e de suporte, agindo conforme as especificidades regionais, com o intuito de conceder uma atenção à saúde mais eficaz, e que abrange profissões e áreas até então não agraciada no âmbito das equipes na ESF, tendo como responsabilidade central atuar e reforçar as diretrizes da $\mathrm{AB}$ (2).

O NASF pode ser composto por profissionais de diversas áreas do conhecimento, partilhando as práticas em saúde nos territórios sob incumbência das equipes da ESF e/ou das Equipes de Atenção Básica
(EqAB) para populações específicas, tais como as ribeirinhas, fluviais ou as que são atendidas em consultórios na rua. Há três modalidades de NASF, sendo eles o 1, 2 e 3 , que divergem quanto à carga horária de trabalho e ao número de equipes ESF/EqAB que assistem. Segundo dados do Departamento de Atenção Básica do Ministério da Saúde (DAB/MS), de junho de 2017, ocorreu a implementação de 4.342 equipes NASF no País, sendo 2.490, 853 e 999 das modalidades 1,2 e 3 , respectivamente (2, $3)$.

No entanto, o trabalho do NASF apenas pode ser realizado com base na organização de momentos relacionais, quando, através de momentos de encontro, suceda troca de saberes/afetos entre os profissionais de diferentes áreas ou setores, visando ampliar a oportunidade de as equipes estabelecerem relações de cooperação, incumbindo-se pelas ações desencadeadas, em um processo de construção de integralidade da atenção. Que pode acabar contribuindo para a superação de um processo de trabalho centralizado nos modelos já existentes e pouco resolutivo, passando a centrar seus esforços nos usuários, ultrapassando a produção de procedimentos em si para a produção de saúde em defesa da vida (3).

A Atenção Básica à Saúde, mais do que ser o principal meio de acesso ao sistema de saúde brasileiro, tem capacidade para resolver cerca de $85 \%$ das demandas de saúde da população. Os NASFs foram idealizados com o intuito de qualificar e ampliar o propósito das ações da $\mathrm{AB}$ com equipes multiprofissionais. Priorizando o trabalho compartilhado com as equipes de Saúde da Família (eSF) e visando transpor a lógica fragmentada, e ainda hegemônica, no cuidado à saúde, com trabalhos pautados de acordo com novas formas de arranjos organizacionais $(2,3)$.

A relevância do presente projeto está associada ao fato que embora se identifique a potencialidade do NASF na resolução e qualificação da $\mathrm{AB}$, ainda são apontadas diversas maneiras para sua implantação e operacionalização; e é descrita pouca compreensão dos profissionais relacionada ao modo de exercer o apoio matricial. Diante do exposto este estudo visa verificar na literatura científica o conhecimento dos profissionais de saúde acerca do NASF. Realizado através de revisão integrada da literatura, o qual tem por finalidade avaliar os conhecimentos referentes ao tema proposto.

\section{MÉTODO}

Trata-se de um estudo bibliográfico, tipo revisão integrativa onde realizou-se a busca de artigos para desenvolvimento metodológico e foram percorridas, as seguintes etapas: elaboração da questão norteadora e objetivo do estudo; definição de critérios de inclusão e exclusão das produções científicas; busca de estudos científicos nas bases de dados e bibliotecas virtuais; análise e categorização das produções encontradas; resultados e discussão dos achados (4). 


\section{artigo}

Siqueira, G. M. C., Silva, L. S. R., Silva, Y. G. C. F., Gomes, P. A. M., Matos, P. B. P., Ferreira, M. S.

Conhecimento dos profissionais de saúde acerca do núcleo de apoio à saúde da família

Para o levantamento da questão norteadora, aplicou-se a estratégia PICo, onde $\mathrm{P}$ = População/Paciente; I = Interesse; e Co $=$ Contexto $(\mathrm{P}-$ Profissionais de saúde; $\mathrm{I}$ - Conhecimento acerca; Co - Núcleo de Apoio à Saúde da Família. Estratégia baseada na segmentação da hipótese, que visa o levantamento de dados de forma sistematizada. Desta forma, definiu-se a seguinte questão norteadora da pesquisa: "Qual é o conhecimento dos profissionais de saúde acerca do NASF?".

Para seleção dos artigos, utilizaram-se como critérios de inclusão: ser artigo original, ter sido publicado em português, inglês ou espanhol, nos últimos dez anos (2009 a 2019) e estar disponível na íntegra. Foram excluídas as literaturas cinzas, bem como publicações repetidas de estudos em mais de uma base de dados e os artigos que não responderam à questão norteadora do estudo.

O levantamento dos dados, que ocorreu durante o mês de janeiro e março de 2020 nas seguintes bases de dados e bibliotecas virtuais: Base de Dados em Enfermagem (BDENF), Medical Literature Analysis and Retrieval System Online (MEDLI$\mathrm{NE}$ ); na Literatura Latino-Americana e do Caribe em Ciências da Saúde (LILACS); e na Biblioteca Virtual Scientific Electronic Library Online (SciELO). Elegendo estas bases de dados e bibliotecas por entender que atingem a literatura publicada, como também referências técnico-científicas brasileiras em saúde pública e coletiva. Foram realizados cruzamentos dos descritores "Atenção Primária à Saúde/Primary Health Care", "Saúde da Família/Family Health", "Estratégia Saúde da Família/Family Health Strategy", presentes na base de Descritores em Ciências da Saúde (DecS) e "Núcleo de Apoio à Saúde da Família", que não consta no DecS mas é pertinente aos objetivos do estudo, combinados com operador booleano AND, efetuando busca conjunta e individual para que possíveis diferenças fossem corrigidas.

A seleção dos estudos, baseou-se no Preferred Reporting Items for Systematic Reviewand Meta-Analyse (PRISMA) um checklist com 27 itens e um fluxograma de

Figura 1: Fluxograma da seleção dos estudos segundo o Preferred Reporting Items for Systematic Reviews and Meta-Analyses (PRISMA 2015). Recife (PE), Brasil, 2020.

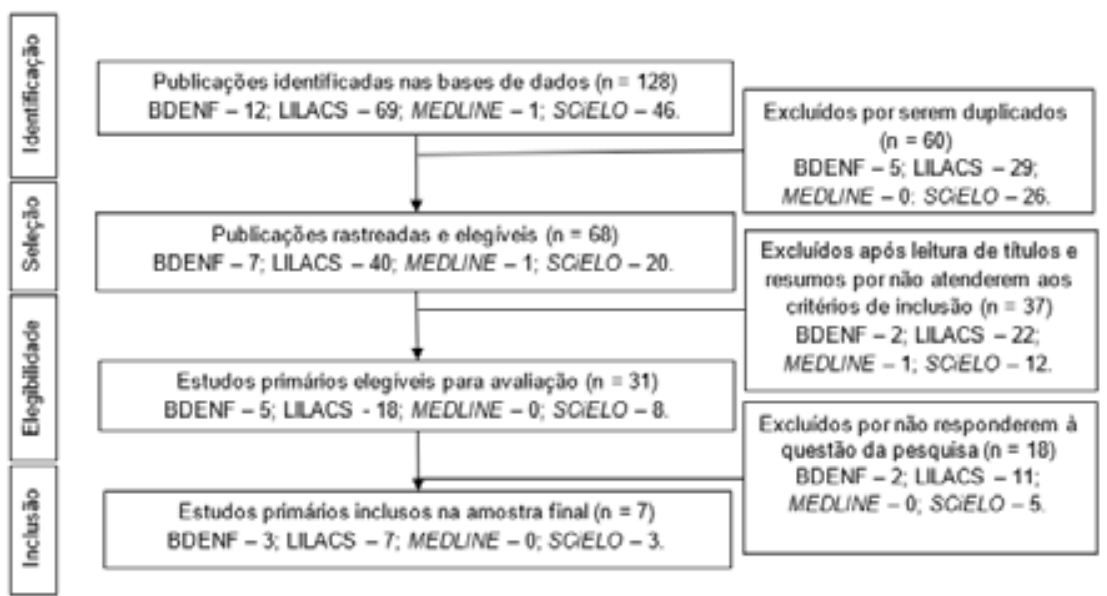

Fonte: Dados da pesquisa, 2020

quatro etapas, com o objetivo de auxiliar no desenvolvimento de artigos (5). A princípio eliminaram-se por meio da leitura de títulos e resumos, estudos duplicados. Destes pré-selecionados, realizou-se leitura na íntegra, a fim de verificar os que atendem à questão norteadora e aos critérios de inclusão/exclusão. Construiu-se então a amostra final com estudos pertinentes aos critérios pré-estabelecidos (Figura 2).

O nível de evidência dos estudos selecionados foi determinado de acordo com a Agency for Healthcare Research and Quality (6): Nível I- Metanálise de múltiplos estudos controlados; Nível II- Estudos individuais com delineamento experimental; Nível III- Estudo com delineamento quase-experimental como estudo sem randomização com grupo único pré e pós-teste, séries temporais ou caso-controle; Nível IV- Estudo com delineamento não-experimental como pesquisa descritiva correlacional e qualitativa ou estudos de caso; Nível V- Relatório de casos ou dado obtido de forma sistemática, de qualidade verificável ou dados de avaliação de programas; e por fim o Nível VI- Opiniões de autoridades respeitáveis baseada na competência clínica ou opinião de comitês de especialistas, incluindo interpretações de informações não baseadas em pesquisas.

Visando simplificar o entendimento das publicações selecionadas nesta revisão integrativa, organizaram-se os dados em figuras e tabelas, expostos de forma descritiva.

\section{RESULTADOS}

Os estudos levantados estão dispostos evidenciando seus títulos, autores, anos de publicação, níveis de evidências, objetivos e resultados. Após a leitura dos artigos selecionados, os estudos foram categorizados, classificando o conhecimento produzido sobre o tema, em níveis de evidência, majoritariamente nível IV- Estudo com delineamento não-experimental como pesquisa descritiva correlacional e qualitativa ou estudos de caso. Os principais achados dispostos nos objetivos e conclusões, estão diretamente associados a interação entre NASF e as EqAB, bem como voltadas aos processos de trabalhos e profissionais que compõem de modo interdisciplinar as equipes do NASF (Quadro 1).

Dado o exposto, é possível observar que embora o raciocínio do NASF-AB demande compreensão mais ampla acerca das práticas de atenção à saúde, essa estratégia de apoio/assistência compartilhada com as ESF está sendo, aos poucos, integrada e valorizada pelos trabalhadores da $\mathrm{AB}$.

\section{DISCUSSÃO}


Quadro 1: Síntese dos principais achados sobre o conhecimento dos profissionais de saúde acerca do Núcleo de Apoio à Saúde da Familia. Recife, Pernambuco (PE), 2020.

N Título/Base

Avaliação do processo de implantação das equipes dos núcleos de apoio à saúde da família/ BDENF.

Evaluation of the multiprofessional work of the family health support center (NASF)/BDENF.
Martiniano et al., (2013).

Brasil

IV

Reis et al. (2017)

Brasil

IV

Núcleo de apoio a saúde da família: atuação da equipe junto à estratégia saúde da família/BDENF.

\section{Santana et al. (2015) Brasil IV}

Analisar a atuação das equipes do NASF junto as Equipes de Saúde da Família e identificar potencialidades para melhoria do processo de trabalho do NASF dentro do município de Cabedelo, Paraíba, Brasil.
A forma tênue do Ministério da Saúde em orientar a implantação dos NASF, aliada ao modelo de gestão de cada município, acaba por estabelecer um modelo de NASF que nem sempre atende à proposta de reorganizador da rede de serviços de saúde.

Compreender os significados atribuídos pelos atores (assistente social, educador físico, farmacêutico, nutricionista e sicólogo) ao trabauma unidade do Núcleo de Apoio à Saúde da Familia.

Destacou-se a necessidade de fortalecer o processo de trabalho do NASF junto a Estratégia de Saúde da Familia, construindo uma ação de interação entre trabalhadores e os usuários, a fim de superar os desafios no processo de trabalho em equipe e buscar alcançar uma meIhor assistência coletiva em saúde.

No entanto, essa atuação diferenciada não se estende às equipes de Saúde da Família vinculadas, salvo em situações específicas de saúde mental, quando há utilização do Apoio Matricial para discussão e condução dos casos apresentados pelas equipes da Estratégia de Saúde da Familia.
Analisar a compreensão dos profissionais da equipe de Saúde Família e do Núcleo de Apoio à Saúde da Familia (NASF) quanto às práticas da terapia ocupacional na APS, como membro da equipe NASF.
Houve dificuldade para diferenciar práticas próprias do terapeuta ocupacional, mesmo quando há compartilhamento de trabalho. Isso sugere a necessidade de implementar estratégias, como o matriciamento, para superação das lacunas no conhecimento quanto às especificidades de cada profissão que atua na APS. 


\section{artigo}

Siqueira, G. M. C., Silva, L. S. R., Silva, Y. G. C. F., Gomes, P. A. M., Matos, P. B. P., Ferreira, M. S.

Conhecimento dos profissionais de saúde acerca do núcleo de apoio à saúde da familia

Avaliação das ações da Fonoaudiologia no NASF da cidade do Recife/LILACS.

Caracterização do trabalho de enfermeiros e profissionais do Núcleo de Apoio à Saúde da Família na Atenção Primária/LILACS.

Núcleo de apoio à saúde da familia: refletindo sobre as acepções emergentes da prática/ LILACS.
Andrade et al. (2014) Brasil IV

Avaliar as ações da Fonoaudiologia nos Núcleos de Apoio à Saúde da Família da cidade do Recife (PE).

Este estudo se propôs caracterizar o trabalho de enfermeiros da Estratégia Saúde da Familia e profissionais de saúde de Núcleos de Apoio à Saúde da Família em um município da Paraíba, Brasil.

Analisar as práticas profissionais das equipes dos Núcleos de Apoio à Saúde da Familia (NASF) dos Sampaio et al. (2013) Brasil IV municípios de Campina Grande e João Pessoa, em consonância com os modelos de apoio matricial identificados.
A Fonoaudiologia tem desempenhado de forma coerente seu papel nos NASF da cidade do Recife. Embora sua atuação seja fundamentada no processo de trabalho, aponta-se a necessidade de intervir em questões relacionadas à estrutura, a fim de aprimorar o desenvolvimento das ações realizadas nesse contexto.

Os profissionais da Atenção Primária à Saúde do município têm que lidar com limitações impostas por um cenário estrutural pouco favorável das unidades de saúde e vínculos trabalhistas marcados por insegurança, falta de solidariedade e baixo reconhecimento.
A assunção de uma ou mais concepções de apoio matricial por uma gestão não ocorre apenas por opção técnica, mas é influenciada por uma variedade de fatores políticos e econômicos que influenciam a adoção de um determinado modelo técnico-assistencial em saúde.
O trabalho do núcleo de apoio à saúde da va de seus trabalhadores/LILACS.

Leite, Denise Fernandes; Oliveira, Maria Amélia de Campos; Nascimento, Débora Dupas Gonçalves do. (2016)
Analisar as percepções dos profissio-

Brasil IV nais do NASF sobre o trabalho que realizam.
De acordo com as percepções dos profissionais, o entendimento do processo de trabalho do NASF ainda não é totalmente claro e apropriado pelos profissionais do próprio NASF, da ESF e nem pelos usuários, fazendo-se necessário uma maior e melhor integração entre eles, com vistas à qualidade e à efetividade do trabalho nesse nivel de atenção. 
Perfil de atendimento de um Núcleo de Apoio à Saúde da Familia na área de reabilitação, Município de Belo Horizonte,

Estado de Minas

Gerais, Brasil, 2009/ LILACS.

Processo de trabalho entre a Equipe de Atenção Básica e o Núcleo de Apoio à Saúde da Família/ LILACS.
Descrever o perfil dos atendimentos realizados pelo Núcleo de Apoio à Saúde da Família (NASF) na área de reabilitação, Regional Barreiro, Belo Horizonte-MG, Brasil.

Analisar, na Paraíba, a organização do processo de trabalho entre o NASF e as EqAB Silva et al. (2017) Brasil IV quanto ao acesso destas às equipes NASF e ao planejamento das ações conjuntas.

Analisar a organização do processo de trabalho do Núcleo de Apoio à Saúde da Família (NASF).

Caracterizar o modo como as ações de apoio matricial estão inseridas no cotidiaações na atenção primária: experiência de profissionais de ESF e NASF/SciELO.

Percepções de usuários e profissionais da saúde da família sobre o Núcleo de Apoio à Saúde da Familia/SciELO.
Aciole, Geovani Gurgel; Oliveira, Dayana Brasil IV Kelly Silva. (2017)
Brasil IV no; e as ferramentas privilegiadas por médicos e enfermeiros da ESF e por especialistas do Nasf.

Explorar a percepção de usuários e profissionais da Estratégia de Saúde da Família sobre o Núcleo de Apoio à Saúde da Família (Nasf).
O perfil de atendimento do NASF demonstrou que essa estratégia contribuiu para a continuidade do cuidado em reabilitação pautado na integralidade e para a promoção do trabalho interdisciplinar na Atenção Primária à Saúde.

Há, entre as EqAB e equipe NASF, um planejamento inicial de ações conjuntas, definição de atribuições e de critérios de fluxo/encaminhamento, mas o planejamento dinâmico tende a ser menos frequente. Quanto ao acesso, há satisfação das EqAB com o apoio que recebem do NASF, mas predominam o encaminhamento por escrito e o agendamento direto como forma de compartilhamento de casos entre as equipes; a satisfação pode estar associada à divisão das demandas na $A B$.

Há necessidade de discussão crítica e repactuação dos processos de trabalho no NASF, com vistas a materializar suas diretrizes, orientadas pelo atendimento às necessidades de saúde da população.

Os resultados indicam que as equipes têm dificuldade em executar ações compartilhadas de apoio matricial.

As percepções sugerem que a proposta é reconhecida e valorizada pela população e que é necessário fortalecer as práticas de educação permanente e apoio matricial entre os profissionais.
A abordagem interdisciplinar é uma premissa que fundamenta todo o trabalho do NASF, que é entendido como uma experiência de colaboração entre profissionais de diferentes disciplinas que reflete a união de conceitos e relacionamento interprofissional que requer integração e uma estreita relação entre conhecimento e prática, possibilitando um olhar holístico para o paciente. Para tal, algumas atitudes entre os profissionais tornam-se ainda mais relevantes, dentre elas o reconhecimento da relevância de toda profissão, respeito profissional, tolerância, aceitação de sugestões que visem a melhoria da ação, além de respeito pelas divergências e competências cabíveis a cada área e sobretudo ética (7-10). 


\section{artigo}

A interação entre esses dois eixos é necessária tanto no momento da troca de informações entre os profissionais e possibilidades de interferências, como também visando a qualidade e veracidade da informação oferecida de um profissional para outro. $\mathrm{O}$ atual método utilizado para interdisciplinaridade é o do NASF que consiste; apenas em simples reuniões das equipes onde podem compartilhar ações, ainda sim desperta a preocupação dos profissionais deste órgão, pois eles acreditam que ainda deve haver uma amplificação nesse ramo $(8$, 11).

O apoio disponibilizado pelo NASF para a ESF objetiva o constante processo de colaboração interprofissional. No entanto, há objeções no matriciamento do apoio que contém interação de profissionais na APS por conta da distinta realidade dos territórios, pela falta de recursos de comunicação, pela escassez de profissionais e de serviços especializados, problemas estruturais. Outro fator que implica na fragmentação do cuidado é a falta do total entendimento do projeto por parte dos profissionais do NASF e da ESF, o que acentua a vulnerabilidade de trabalhos interdisciplinares (9, 10).

O NASF busca a formação de novos profissionais capacitados na primordial atenção à saúde ampliando o campo de ensino da medicina e da enfermagem além da terapia ocupacional, fisioterapia e fonoaudiologia que são profissões não muito exploradas pelo SUS. Desse modo, cai sobre o NASF juntamente aos gestores a obrigação de discutir sobre o auxílio necessário aos problemas citados acima relacionados a qualidade e quantidade dos profissionais, e a comunicação que requer um maior investimento por ser o meio em que há o compartilhamento tanto de informações profissionais quanto de informações e resoluções de problemas interpessoais (10-14).

Por meio das estratégias de busca encontrou-se uma amostra pequena e pouca disponibilidade de artigos científicos para comparação dos resultados. Assim como apareceram poucos artigos como resultado dos descritores, poucos atenderam ao objetivo do estudo. Ainda, os estudos incluídos apresentam limitações como: unicêntricos, diferentes sistemas de comparação, tamanho pequeno da amostra e falta de randomização.

Assim, não foi possível comprovar a existência de evidência científica relacionada ao conhecimento dos profissionais de saúde acerca do NASF. Fazendo-se necessário a realização de mais estudos contendo uma amostra maior e possibilitando discussão acerca do conhecimento dos profissionais de saúde acerca do NASF.

Podendo contribuir ao disseminar a importância do conhecimento dos profissionais de saúde acerca do NASF. Ampliando a consciência da profissão e auxiliando na capacitação da equipe multiprofissional em saúde.

\section{CONCLUSÃO}

Constatou-se que os profissionais de saúde compreendem a estrutura do NASF, ainda que com algum déficit de informação, declarando o serviço como de extrema importância, apresentando satisfação acerca das atividades realizadas, categorizando como serviço alcançável pela população, sendo está assegurada pelo NASF nos seus direitos na saúde pública. Diante da característica progressista, dos desafios determinados pela situação da saúde no país e das perspectivas associadas aos NASF, faz-se necessário a elaboração de estudos que tratem sobre essa questão e favoreçam a ampliação dos debates acerca da concepção, do funcionamento, do conhecimento e da avaliação do impacto das ações do NASF na qualidade do cuidado.

\section{REFERÊNCIAS}

1. Rocardo D, Andrade CLT, Fausto MCR, Lima SML. Núcleo de Apoio à Saúde da Família (Nasf): panorama nacional a partir de dados do pmaq. Saúde em Debate, 2018; 42 (1): 130-144, set. 2018. http:// doi.org/10.1590/0103-11042018s109

2. Oliveira KS, Baduy R, Melchior R. 0 encontro entre Núcleo de Apoio à Saúde da Família e as equipes de Saúde da Família: a produção de um coletivo cuidador. Physis: Revista de Saúde Coletiva, 2019; 29 (4):1-20. http://doi.org/10.1590/s0103-73312019290403

3. Seus T, Silveira DS, Tomasi E, Thumé E, Facchini LA, Siqueira FV. Estrutura para o trabalho e composição de equipes do Núcleo de Apoio à Saúde da Família: pesquisa nacional - Programa de Melhoria do Acesso e da Qualidade (PMAQ), 2013*. Epidemiologia e Serviços de Saúde, 2020; 28(3):1-11. http://doi.org/10.5123/s167949742019000300017.

4. Lisboa MT. Elementos para elaboração de um desenho de pesquisa | Elements to formulate a research design. Mural Internacional, 2019; 10:38439-1. http://doi.org/10.12957/rmi.2019.38439

5. Barbosa FT, Lira AB, Oliveira Neto OB, Santos LL, Santos IO, Barbosa LT et al. Tutorial para execução de revisões sistemáticas e metaná- lises com estudos de intervenção em anestesia. Brazilian Journal Of Anesthesiology, 2019; 69(3): 299-306. http://doi.org/10.1016/j. bjan.2018.11.007

6. AHRQ. AGENCY FOR HEALTH CARE RESEARCH AND QUALITY (Rockville) (Org.). Quality Improvement and monitoring at your fingertips. 2016. Available from: https://www.qualityindicators.ahrq. gov/

7. Reis ML, Medeiros M, Pacheco LR, Caixeta CC. Evaluation Of The Multiprofessional Work Of The Family Health Support Center (NASF). Texto \& Contexto - Enfermagem, 2016; 25(1):1-9. http://doi. org/10.1590/0104-070720160002810014

8. Nascimento DDG, Quevedo MP, Oliveira MAC. Pleasure At Work In A Family Health Support Team: a dejourian analysis. Texto \& Contexto - Enfermagem, 2017; 26(1). http://doi.org/10.1590/010407072017006380015

9. Previato GF, Baldissera VDA. Retratos da prática interprofissional colaborativa nas equipes da atenção primária à saúde. Rev Gaúcha Enferm. 2018;39:e2017-0132. https://doi.org/10.1590/19831447.2018.2017-0132 


\section{REFERÊNCIAS}

10. Maffissoni AL, Silva KJ, Vendruscolo C,Trindade LL, Metelski FK. Função matriciadora dos Núcleos Ampliados de Saúde da Família: uma revisão integrativa da literatura. : uma revisão integrativa da literatura. Saúde em Debate, 2018; 42(119): 1012-1023. http://doi. org/10.1590/0103-1104201811918

11. Aciole GG, Oliveira DKS. Percepções de usuários e profissionais da saúde da família sobre o Núcleo de Apoio à Saúde da Família. Saúde em Debate, 2017; 41(115):1090-1101.http://doi. org/10.1590/0103-1104201711508

12. Souza TS, Medina MG. Nasf: fragmentação ou integração do trabalho em saúde na aps?. : fragmentação ou integração do trabalho em saúde na APS?. Saúde em Debate, 2018; 42(2):145-158. http://

\section{doi.org/10.1590/0103-11042018s210}

13. Santos FBS, Souza SS, Nascimento GS, Almeida IN. Fisioterapeuta e NASF: conhecimentos de graduados da primeira e segunda década do século XXI. SaudColetiv (Barueri), 2021;11(64):5628-39. https://doi.org/10.36489/saudecoletiva.2021v11i64p5628-5639

14. Amaral IBST, Rodrigues MA, Éden FBS, Silva CSSL, Pinto AA. Apoio institucional: reflexões sobre um novo componente do arranjo organizacional de gestão em saúde. Nursing, 2019;22(249):2663-7. https://doi.org/10.36489/nursing.2019v22i249p2663-2667 